\title{
OPEN Comparison of COVID-19 outcomes among shielded and non-shielded populations
}

\author{
Bhautesh D. Jani ${ }^{1,6}$, Frederick K. Ho ${ }^{1,6}$, David J. Lowe ${ }^{2}$, Jamie P. Traynor ${ }^{2}$, \\ Sean P. MacBride-Stewart ${ }^{3}$, Patrick B. Mark ${ }^{4}$, Frances S. Mair ${ }^{1}$ \& Jill P. Pell ${ }^{5 凶}$
}

Many western countries used shielding (extended self-isolation) of people presumed to be at high-risk from COVID-19 to protect them and reduce healthcare demand. To investigate the effectiveness of this strategy, we linked family practitioner, prescribing, laboratory, hospital and death records and compared COVID-19 outcomes among shielded and non-shielded individuals in the West of Scotland. Of the 1.3 million population, 27,747 (2.03\%) were advised to shield, and 353,085 (26.85\%) were classified a priori as moderate risk. COVID-19 testing was more common in the shielded (7.01\%) and moderate risk (2.03\%) groups, than low risk (0.73\%). Referent to low-risk, the shielded group had higher confirmed infections (RR 8.45, 95\% 7.44-9.59), case-fatality (RR 5.62, 95\% Cl 4.47-7.07) and population mortality (RR $57.56,95 \%$ 44.06-75.19). The moderate-risk had intermediate confirmed infections (RR 4.11, 95\% Cl 3.82-4.42) and population mortality (RR 25.41, 95\% Cl 20.36-31.71) but, due to their higher prevalence, made the largest contribution to deaths (PAF 75.30\%). Age $\geq 70$ years accounted for $49.55 \%$ of deaths. In conclusion, in spite of the shielding strategy, high risk individuals were at increased risk of death. Furthermore, to be effective as a population strategy, shielding criteria would have needed to be widely expanded to include other criteria, such as the elderly.

Early in the COVID-19 pandemic, a major concern was that the demand on health services would exceed capacity in terms of hospitalisations, intensive care unit (ICU) admissions, and ventilation ${ }^{1}$; hence, policy-makers sought interventions that could flatten the curve in severe cases to avoid hospitals becoming overwhelmed. It was assumed that sub-groups of the population would have worse prognosis and, therefore, contribute disproportionately to adverse outcomes and healthcare demands ${ }^{2}$.

Asian countries could implement case and contact finding ${ }^{3}$. Early, widespread 'test, trace, isolate' strategies were made possible by their higher testing capacity and greater willingness to monitor and enforce compliance. In contrast, Europe and the USA were obliged to rely more heavily on non-pharmaceutical interventions in the first wave of the pandemic ${ }^{3}$; general measures, such as physical distancing, face coverings, hand hygiene and lock-downs, designed to reduce transmission in the population as a whole, supplemented by shielding of those assumed to be at higher risk. Notably, Sweden, an outlier in not applying lock-down, nonetheless mandated shielding ${ }^{4}$.

In the UK, a Vulnerable Patient List (Supplementary Table S1) ${ }^{5}$ was produced comprising two categories labelled high risk, highest risk or clinically extremely vulnerable and moderate risk, at risk or clinically vulnerable by various UK organisations. In this manuscript, they are referred to as shielded and moderate risk respectively, with the remaining population labelled low-risk. The shielded group received individual letters strongly recommending they self-isolate over a protracted period; not leaving their homes and avoiding non-essential contact with household members. In Scotland, the shielded group was offered support through a number of interventions; for example, 53\% signed up to have food support, including home delivery of free food boxes and priority food delivery ${ }^{6}$. Additional local schemes were set up to provide home delivery of medicines ${ }^{6}$. Individuals in the shielded group were also eligible to apply for Statutory Sick Pay. In contrast, the moderate risk category was simply advised to be vigilant in adhering to general advice, for example, using hand sanitisers, wearing a face covering, and maintaining 2 -m distance when entering indoor public spaces.

\footnotetext{
${ }^{1}$ Institute of Health and Wellbeing, University of Glasgow, Glasgow G12 9LX, UK. ${ }^{2}$ Queen Elizabeth University Hospital, NHS Greater Glasgow and Clyde, Glasgow G52 4TF, UK. ${ }^{3}$ Pharmacy Services, NHS Greater Glasgow and Clyde, Glasgow G76 7AT, UK. "Institute of Cardiovascular and Medical Sciences, University of Glasgow, Glasgow G12 8TA, UK. ${ }^{5}$ Institute of Health and Wellbeing, University of Glasgow, 1 Lilybank Gardens, Glasgow G12 8RZ, UK. ${ }^{6}$ These authors contributed equally: Bhautesh D. Jani and Frederick K. Ho. ${ }^{\square}$ email: Jill.pell@ glasgow.ac.uk
} 
The category definitions were based largely on expert opinion informed by our understanding of previous viruses and the need for better definitions has been highlighted and discussed ${ }^{7,8}$. Studies are emerging of the risk factors associated with COVID-19 outcomes. Among two million UK community-based app users selfreported heart disease, kidney disease, lung disease, diabetes and obesity were associated with self-reported hospital admission and respiratory support for COVID-199. Similarly, linkage of family practitioner records of 17 million people in England reported a wide range of long-term conditions associated with in-hospital death from COVID-19 including: respiratory, heart, liver and kidney disease, diabetes, cancers, stroke and organ transplantation ${ }^{10}$. Unfortunately, the investigators did not have access to deaths in the community. COVID-19 risk scores are being developed in an attempt to improve identification of high risk individuals who could be advised to shield ${ }^{11}$ but attempts to investigate the potential contribution of a shielding strategy to population-level outcomes and healthcare demands have so far been largely limited to mathematical modelling ${ }^{12-20}$.

The aims of this study were to compare those classified, a priori, as high risk (and therefore advised to shield) and those classified as moderate and low-risk, in terms of their actual risk of COVID-19 infection and outcomes and the extent to which they accounted for COVID-19 related outcomes at a population level.

\section{Results}

Of the 1,315,071 people registered with family practitioners in NHS Greater Glasgow and Clyde in the West of Scotland, 26,747 (2.03\%) were on the shielding list and 353,085 (26.85\%) were classified, a priori, as moderaterisk. Of the 26,747 shielded group, 18,147 (55.78\%) had severe respiratory disease, $5349(16.44 \%)$ were on immunosuppressive therapies, $2491(7.66 \%)$ had specific cancers, $1245(3.83 \%)$ had received organ transplants, 475 (1.78\%) were on renal dialysis, and less than five were pregnant and had severe heart disease. Of the 353,085 classified as moderate-risk, 160,215 (45.38\%) had hypertension, 151,865 (43.01\%) had chronic lung disease, $139,568(39.53 \%)$ were $\geq 70$ years of age, $64,358(18.23 \%)$ had diabetes, $48,571(13.81 \%)$ had heart disease, and $1195(0.34 \%)$ had a weakened immune system.

Shielded and moderate-risk categories. Overall, 15,865 (1.21\%) people were tested for COVID-19. The likelihood of being tested increased with age, was higher in women and the moderate-risk category and highest in the shielded group (Table 1). Overall, 3348 (0.25\%) people had confirmed COVID-19 infection. The likelihood of laboratory-confirmed COVID-19 infection followed similar patterns as testing. It increased with age, was higher in women, was highest in the shielded group and lowest in the low-risk category (Table 2). After adjustment for sex and deprivation quintile, the risk of laboratory-confirmed infection remained higher in the moderate-risk category and highest in the shielded group (Fig. 1 and Supplementary Table S2).

Overall, 1661 people were hospitalised for COVID-19. Within the general population, hospitalisations increased with age but were comparable between men and women (Table 2). Hospitalisations were more common in the moderate-risk category and most common in the shielded group (Table 2), remaining so after adjustment for sex and deprivation (Fig. 1 and Supplementary Table S2). Overall, 122 people were admitted to ICU wards for COVID-19. ICU admissions were significantly more common among people aged 45-64 years of age than among older people (Table 2). Compared with the low-risk category, the shielded group were 18 times more likely to be hospitalised but only 4 times more likely to be admitted to ICU (Fig. 1 and Supplementary Table S2). Overall, 1027 (0.08\%) people died from COVID-19. Within the general population, mortality increased with age but was similar in men and women (Table 2). Population mortality was higher in the moderate-risk category and highest in the shielded group (Table 2) and remained so after adjustment for sex and deprivation (Fig. 1 and Supplementary Table S2). Mortality in the community before/without admission to hospital was higher in the moderate-risk category $\left(\mathrm{RR}_{\text {moderate/low }}[95 \% \mathrm{CI}] 79.7\right.$ [39.9-159.0], $\left.p<0.0001\right)$ and highest in the shielded group $\left(\mathrm{RR}_{\text {shielded/low }}[95 \% \mathrm{CI}] 182.1\right.$ [86.5-383.3], $\left.p<0.0001\right)$. A similar pattern was observed for mortality before/ without ICU admission $\left(\mathrm{RR}_{\text {moderate/low }}[95 \% \mathrm{CI}] 40.8\right.$ [29.0-57.4], $p<0.0001 ; \mathrm{RR}_{\text {shielded/low }}[95 \% \mathrm{CI}] 120.8$ [83.0, 175.9], $p<0.0001)$.

Among the sub-group with laboratory-confirmed (test-positive) COVID-19 infection, 1661 (49.6\%) were hospitalised. Hospitalisations increased with age but were comparable between men and women (Table 3). The moderate-risk category was more likely to be hospitalised and the shielded group most likely (Table 3), remaining so after adjustment for age and deprivation (Fig. 2 and Supplementary Table S3). Among those with laboratoryconfirmed infection, ICU admissions were more common in men and more common in people aged 45-64 years than those older (Table 3). Low-risk cases were more likely to be admitted to ICU than the moderate-risk and shielded groups (Fig. 2). Among the sub-group with clinically-confirmed (test-positive or COVID-19 related death) COVID-19 infection, 1027 (26.70\%) died (Table 3). Case-fatality increased by age and was higher in men than women. It was lowest in the low-risk category but not significantly different between the moderate-risk and shielded groups $\left(\mathrm{RR}_{\text {shielded/moderate }}[95 \% \mathrm{CI}] 1.12\right.$ [0.96-1.31], $\left.p=0.14\right)$ (Fig. 2 and Supplementary Table S3).

The shielded group accounted for $7.62 \%$ of laboratory-confirmed COVID-19 infections, $12.70 \%$ of COVID-19 hospitalisations, $2.69 \%$ of ICU admissions and $13.22 \%$ of COVID-19 related deaths (Supplementary Table S4). The corresponding figures for the moderate-risk category were $42.06 \%, 53.28 \%, 22.96 \%$ and $75.30 \%$. To prevent at least $80 \%$ of deaths, $28.8 \%$ of the population would have had to receive the current level of shielding including those with five criteria classified as moderate- risk at the time of the study (Supplementary Figure S1).

Individual risk criteria. Due to insufficient numbers, the individual risk criteria models could not be run for pregnant women with severe heart disease or for COVID-19 related ICU admission in the shielded category. All the remaining individual risk criteria were associated with higher likelihood of being tested for COVID-19 (Table 1), laboratory-confirmed infection (Table 2), hospitalisation, population mortality (Fig. 1 and Supplementary Table S2) and case-fatality (Fig. 2 and Supplementary Table S3) independent of sex and deprivation. 


\begin{tabular}{|c|c|c|c|}
\hline & \multicolumn{2}{|c|}{ COVID-19 testing status } & \multirow[b]{4}{*}{$p$ value } \\
\hline & \multirow{3}{*}{$\begin{array}{l}\text { Not tested } \\
\mathrm{N}=1,299,206 \\
\mathrm{n}(\%)\end{array}$} & \multirow{3}{*}{\begin{tabular}{|l|} 
Tested \\
$\mathrm{N}=15,865$ \\
$\mathrm{n}(\%)$ \\
\end{tabular}} & \\
\hline & & & \\
\hline & & & \\
\hline Age group (years) & & & $<0.0001$ \\
\hline $0-24$ & $355,238(99.49)$ & $1822(0.51)$ & \\
\hline $25-44$ & $410,408(99.22)$ & $3247(0.78)$ & \\
\hline $45-64$ & $340,268(98.65)$ & $4660(1.35)$ & \\
\hline$\geq 65$ & $193,292(96.92)$ & $6136(3.08)$ & \\
\hline Sex & & & $<0.0001$ \\
\hline Male & $654,041(99.01)$ & $6569(0.99)$ & \\
\hline Female & $645,165(98.58)$ & $9296(1.42)$ & \\
\hline Deprivation quintile & & & $<0.0001$ \\
\hline 1 (most deprived) & $461,672(98.67)$ & $6211(1.33)$ & \\
\hline 2 & $230,402(98.75)$ & $2921(1.25)$ & \\
\hline 3 & $194,702(98.90)$ & $2175(1.10)$ & \\
\hline 4 & $173,456(98.93)$ & $1883(1.07)$ & \\
\hline 5 (most affluent) & $238,974(98.89)$ & $2675(1.11)$ & \\
\hline Risk category & & & $<0.0001$ \\
\hline Low & $928,420(99.27)$ & $6819(0.73)$ & \\
\hline Moderate & $345,913(97.97)$ & $7172(2.03)$ & \\
\hline Shielded & $24,873(92.99)$ & $1874(7.01)$ & \\
\hline \multicolumn{4}{|l|}{ Moderate risk criteria } \\
\hline Chronic respiratory disease & $149,325(98.33)$ & $2540(1.67)$ & $<0.0001$ \\
\hline Heart disease & $46,728(96.21)$ & $1843(3.79)$ & $<0.0001$ \\
\hline Hypertension & $156,286(97.55)$ & $3929(2.45)$ & $<0.0001$ \\
\hline Diabetes & $62,482(97.09)$ & $1876(2.91)$ & $<0.0001$ \\
\hline Weakened immune system & $1140(95.40)$ & $55(4.60)$ & $<0.0001$ \\
\hline$\geq 70$ years of age & $134,305(96.23)$ & $5263(3.77)$ & $<0.0001$ \\
\hline \multicolumn{4}{|l|}{ Shielded criteria } \\
\hline Severe respiratory disease & $17,146(94.48)$ & $1001(5.52)$ & $<0.0001$ \\
\hline Specific cancers & $2075(83.30)$ & $416(16.70)$ & $<0.0001$ \\
\hline Pregnant with severe heart disease & $<5$ & 0 & - \\
\hline Immunosuppressive therapy & $5028(94.00)$ & $321(6.00)$ & $<0.0001$ \\
\hline Solid organ transplant & $1149(92.29)$ & $96(7.71)$ & $<0.0001$ \\
\hline Rare diseases and inborn errors of metabolism & $1623(91.95)$ & $142(8.05)$ & $<0.0001$ \\
\hline Renal dialysis & $305(64.21)$ & $170(35.79)$ & $<0.0001$ \\
\hline
\end{tabular}

Table 1. COVID-19 testing status by sociodemographic characteristics, risk category and risk criteria. $\mathrm{N}$ number.

Among the moderate-risk category criteria, age $\geq 70$ years and weakened immune system had risks of population mortality (Fig. 1 and Supplementary Table S2) and case-fatality (Fig. 2 and Supplementary Table S3) at least as high as the overall shielded group. Apart from the $0.13 \%$ of people with relevant rare diseases or inborn errors of metabolism and $1.78 \%$ on renal dialysis, the strongest associations were observed for those aged $\geq 70$ years who were eight times as likely to have confirmed infection (Fig. 1 and Supplementary Table S2); seven times as likely to die following confirmed infection (Fig. 2 and Supplementary Table S3); and 74 times as likely to die overall (Fig. 1 and Supplementary Table S2) compared with the low-risk category. Being $\geq 70$ years of age accounted for $17.81 \%$ of confirmed COVID-19 infections, $22.19 \%$ of COVID-19 related hospitalisations, and $49.55 \%$ of COVID-19 related deaths (Supplementary Table S4). Among those hospitalised for COVID-19, the likelihood of ICU admission was significantly lower for all individual risk criteria in the moderate-risk category, other than diabetes (Fig. 2 and Supplementary Table S3). In particular, hospitalised patients $\geq 70$ years of age were 14 times less likely to be admitted to ICU than low-risk hospitalised patients (Fig. 2 and Supplementary Table S3).

\section{Discussion}

The $2.03 \%$ of people advised to shield were, nonetheless, eight times more likely to have confirmed infections than the low-risk category, five times more likely to die following confirmed infection and 49 times more likely to die from COVID-19 overall. Whilst selective testing might explain the first outcome, it does not explain higher overall mortality which suggests that the shielding strategy was not as effective as was hoped. 


\begin{tabular}{|c|c|c|c|c|c|c|c|c|c|c|c|c|}
\hline & \multicolumn{3}{|c|}{ Confirmed COVID-19 infection } & \multicolumn{3}{|c|}{ COVID-19 hospitalisation } & \multicolumn{3}{|c|}{ COVID-19 ICU admission } & \multicolumn{3}{|c|}{ COVID-19 mortality } \\
\hline & \multirow{3}{*}{\begin{tabular}{|l|}
$\begin{array}{l}\text { Negative test/ } \\
\text { Not tested }\end{array}$ \\
$\mathrm{N}=1,311,723$ \\
$\mathrm{n}(\%)$ \\
\end{tabular}} & \multirow{3}{*}{\begin{tabular}{|l|} 
Positive test \\
$\mathrm{N}=\mathbf{3 , 3 4 8}$ \\
$\mathrm{n}(\%)$
\end{tabular}} & \multirow[b]{3}{*}{$p$ value } & \multirow{3}{*}{\begin{tabular}{|l} 
Not admitted \\
$\mathrm{N}=1,313,410$ \\
$\mathrm{n}(\%)$
\end{tabular}} & \multirow{3}{*}{\begin{tabular}{|l|} 
Admitted \\
$\mathrm{N}=1661$ \\
$\mathrm{n}(\%)$ \\
\end{tabular}} & \multirow[b]{3}{*}{$p$ value } & \multirow{3}{*}{\begin{tabular}{|l|} 
Not admitted \\
$\mathrm{N}=1,314,949$ \\
$\mathrm{n}(\%)$
\end{tabular}} & \multirow{3}{*}{\begin{tabular}{|l} 
Admitted \\
$\mathrm{N}=122$ \\
$\mathbf{n}(\%)$
\end{tabular}} & \multirow[b]{3}{*}{$p$ value } & \multirow{3}{*}{\begin{tabular}{|l} 
Alive \\
$\mathrm{N}=1,314,044$ \\
$\mathrm{n}(\%)$
\end{tabular}} & \multirow{3}{*}{\begin{tabular}{|l|} 
Dead \\
$\mathrm{N}=1027$ \\
$\mathrm{n}(\%)$ \\
\end{tabular}} & \multirow[b]{3}{*}{$p$ value } \\
\hline & & & & & & & & & & & & \\
\hline & & & & & & & & & & & & \\
\hline $\begin{array}{l}\text { Age group } \\
\text { (years) }\end{array}$ & & & $<0.0001$ & & & $<0.0001$ & & & 0.0005 & & & $<0.0001$ \\
\hline $0-24$ & \begin{tabular}{|l}
356,944 \\
$(99.97)$
\end{tabular} & $116(0.03)$ & & \begin{tabular}{|l|}
357,041 \\
$(99.99)$
\end{tabular} & $19(0.01)$ & & \begin{tabular}{|l|}
357,060 \\
$(100.00)$
\end{tabular} & 0 & & $\begin{array}{l}357,060 \\
(100.00)\end{array}$ & $0(0.00)$ & \\
\hline $25-44$ & \begin{tabular}{|l|}
413,123 \\
$(99.87)$
\end{tabular} & $532(0.13)$ & & \begin{tabular}{|l|}
413,532 \\
$(99.97)$
\end{tabular} & $123(0.03)$ & & \begin{tabular}{|l|}
413,643 \\
$(100.00)$
\end{tabular} & $12(0.00)$ & & \begin{tabular}{|l|}
413,647 \\
$(100.00)$
\end{tabular} & $8(0.00)$ & \\
\hline $45-64$ & $\begin{array}{l}343,856 \\
(99.69)\end{array}$ & $1072(0.31)$ & & \begin{tabular}{|l|}
344,409 \\
$(99.85)$
\end{tabular} & $519(0.15)$ & & $\begin{array}{l}344,846 \\
(99.98)\end{array}$ & $82(0.02)$ & & \begin{tabular}{|l|}
344,838 \\
$(99.97)$
\end{tabular} & $90(0.03)$ & \\
\hline$\geq 65$ & $\begin{array}{l}197,800 \\
(99.18)\end{array}$ & $1628(0.82)$ & & $\begin{array}{l}198,428 \\
(99.50)\end{array}$ & $1000(0.50)$ & & $\begin{array}{l}199,400 \\
(99.99)\end{array}$ & $28(0.01)$ & & $\begin{array}{l}198,499 \\
(99.53)\end{array}$ & $929(0.47)$ & \\
\hline \multicolumn{13}{|l|}{ Sex } \\
\hline Male & \begin{tabular}{|l|}
659,203 \\
$(99.79)$
\end{tabular} & $1407(0.21)$ & $<0.0001$ & \begin{tabular}{|l|}
659,781 \\
$(99.87)$
\end{tabular} & $829(0.13)$ & 0.81 & $\begin{array}{l}660,525 \\
(99.99)\end{array}$ & $85(0.01)$ & $<0.0001$ & \begin{tabular}{|l|}
660,092 \\
$(99.92)$
\end{tabular} & $518(0.08)$ & 0.92 \\
\hline Female & $\begin{array}{l}652,250 \\
(99.70)\end{array}$ & $1941(0.30)$ & & \begin{tabular}{|l|}
659,369 \\
$(99.87)$
\end{tabular} & $832(0.13)$ & & \begin{tabular}{|l|}
660,908 \\
$(99.99)$
\end{tabular} & $37(0.01)$ & & \begin{tabular}{|l|}
653,952 \\
$(99.92)$ \\
\end{tabular} & $509(0.08)$ & \\
\hline $\begin{array}{l}\text { Deprivation } \\
\text { quintile }\end{array}$ & & & 0.0002 & & & $<0.0001$ & & & 0.18 & & & $<0.0001$ \\
\hline $\begin{array}{l}1 \text { (most } \\
\text { deprived) }\end{array}$ & \begin{tabular}{|l|}
466,582 \\
$(99.72)$
\end{tabular} & $1301(0.28)$ & & \begin{tabular}{|l|}
467,146 \\
$(99.84)$
\end{tabular} & $737(0.16)$ & & $\begin{array}{l}467,832 \\
(99.99)\end{array}$ & $51(0.01)$ & & \begin{tabular}{|l|}
467,442 \\
$(99.91)$
\end{tabular} & $441(0.09)$ & \\
\hline 2 & $\begin{array}{l}232,710 \\
(99.74)\end{array}$ & $613(0.26)$ & & $\begin{array}{l}233,041 \\
(99.88)\end{array}$ & $282(0.12)$ & & $\begin{array}{l}233,302 \\
(99.99)\end{array}$ & $21(0.01)$ & & $\begin{array}{l}233,181 \\
(99.94)\end{array}$ & $142(0.06)$ & \\
\hline 3 & $\begin{array}{l}196,416 \\
(99.77)\end{array}$ & $461(0.23)$ & & $\begin{array}{l}196,651 \\
(99.89)\end{array}$ & $226(0.11)$ & & $\begin{array}{l}196,854 \\
(99.99)\end{array}$ & $23(0.01)$ & & $\begin{array}{l}196,724 \\
(99.92)\end{array}$ & $153(0.08)$ & \\
\hline 4 & $\begin{array}{l}174,939 \\
(99.77)\end{array}$ & $400(0.23)$ & & $\begin{array}{l}175,143 \\
(99.89)\end{array}$ & $196(0.11)$ & & $\begin{array}{l}175,329 \\
(99.99)\end{array}$ & $10(0.01)$ & & $\begin{array}{l}175,209 \\
(99.93)\end{array}$ & $130(0.07)$ & \\
\hline $\begin{array}{l}5 \text { (most } \\
\text { affluent) }\end{array}$ & $\begin{array}{l}241,076 \\
(99.76)\end{array}$ & $573(0.24)$ & & $\begin{array}{l}241,429 \\
(99.91)\end{array}$ & $220(0.09)$ & & $\begin{array}{l}241,632 \\
(99.99)\end{array}$ & $17(0.01)$ & & $\begin{array}{l}241,488 \\
(99.93)\end{array}$ & $161(0.07)$ & \\
\hline $\begin{array}{l}\text { Risk cat- } \\
\text { egory }\end{array}$ & & & $<0.0001$ & & & $<0.0001$ & & & $<0.0001$ & & & $<0.0001$ \\
\hline Low & $\begin{array}{l}934,049 \\
(99.87)\end{array}$ & $1190(0.13)$ & & $\begin{array}{l}93,4839 \\
(99.96)\end{array}$ & $400(0.04)$ & & \begin{tabular}{|l|}
935,174 \\
$(99.99)$
\end{tabular} & $65(0.01)$ & & \begin{tabular}{|l|}
935,155 \\
$(99.99)$
\end{tabular} & $84(0.01)$ & \\
\hline Moderate & $\begin{array}{l}351,226 \\
(99.47)\end{array}$ & $1859(0.53)$ & & $\begin{array}{l}35,2054 \\
(99.71)\end{array}$ & $1031(0.29)$ & & $\begin{array}{l}353,033 \\
(99.99)\end{array}$ & $52(0.01)$ & & \begin{tabular}{|l|}
352,282 \\
$(99.77)$
\end{tabular} & $803(0.23)$ & \\
\hline Shielded & $26,448(98.88)$ & $299(1.12)$ & & $26,517(99.14)$ & $230(0.86)$ & & 26,742 (99.98) & $5(0.02)$ & & $26,607(99.48)$ & $140(0.52)$ & \\
\hline \multicolumn{13}{|c|}{ Moderate risk criteria } \\
\hline $\begin{array}{l}\text { Chronic } \\
\text { respiratory } \\
\text { disease }\end{array}$ & $\begin{array}{l}151,414 \\
(99.70)\end{array}$ & $451(0.30)$ & 0.0005 & $\begin{array}{l}151,618 \\
(99.84)\end{array}$ & $247(0.16)$ & $<0.0001$ & $\begin{array}{l}151,853 \\
(99.99)\end{array}$ & $12(0.01)$ & 0.65 & $\begin{array}{l}151,812 \\
(99.97)\end{array}$ & $53(0.03)$ & $<0.0001$ \\
\hline $\begin{array}{l}\text { Heart } \\
\text { disease }\end{array}$ & $48,176(99.19)$ & $395(0.81)$ & $<0.0001$ & $48,325(99.49)$ & $246(0.51)$ & $<0.0001$ & 48,564 (99.99) & $7(0.01)$ & 0.34 & $48,456(99.76)$ & $115(0.24)$ & $<0.0001$ \\
\hline $\begin{array}{l}\text { Hyperten- } \\
\text { sion }\end{array}$ & $\begin{array}{l}159,267 \\
(99.41)\end{array}$ & $948(0.59)$ & $<0.0001$ & $\begin{array}{l}159,670 \\
(99.66)\end{array}$ & $545(0.34)$ & $<0.0001$ & $\begin{array}{l}160,189 \\
(99.98)\end{array}$ & $26(0.02)$ & 0.003 & $\begin{array}{l}159,991 \\
(99.86)\end{array}$ & $224(0.14)$ & $<0.0001$ \\
\hline Diabetes & 63,903 (99.29) & $455(0.71)$ & $<0.0001$ & 64,063 (99.54) & $295(0.46)$ & $<0.0001$ & \begin{tabular}{|l|}
$64,335(99.96)$ \\
\end{tabular} & $23(0.04)$ & $<0.0001$ & 64,263 (99.85) & $95(0.15)$ & $<0.0001$ \\
\hline $\begin{array}{l}\text { Weakened } \\
\text { immune } \\
\text { system }\end{array}$ & $1183(99.00)$ & $12(1.00)$ & $<0.0001$ & $1185(99.16)$ & $10(0.84)$ & $<0.0001$ & $1195(100.00)$ & $0(0.00)$ & - & $1189(99.50)$ & $6(0.50)$ & $<0.0001$ \\
\hline $\begin{array}{l}\geq 70 \text { years } \\
\text { of age }\end{array}$ & $\begin{array}{l}138,115 \\
(98.96)\end{array}$ & $1,453(1.04)$ & $<0.0001$ & $\begin{array}{l}138,701 \\
(99.38)\end{array}$ & $867(0.62)$ & $<0.0001$ & $\begin{array}{l}139,560 \\
(99.99)\end{array}$ & $8(0.01)$ & 0.19 & $\begin{array}{l}138,690 \\
(99.37)\end{array}$ & $878(0.63)$ & $<0.0001$ \\
\hline \multicolumn{13}{|c|}{ Shielded group } \\
\hline $\begin{array}{l}\text { Severe } \\
\text { respiratory } \\
\text { disease }\end{array}$ & 17,981 (99.09) & $166(0.91)$ & $<0.0001$ & $18,012(99.26)$ & $135(0.74)$ & $<0.0001$ & 18,146 (99.99) & $<5$ & - & $18,059(99.52)$ & $88(0.48)$ & $<0.0001$ \\
\hline $\begin{array}{l}\text { Specific } \\
\text { cancers }\end{array}$ & 2452 (98.43) & $39(1.57)$ & $<0.0001$ & $2462(98.84)$ & $29(1.16)$ & $<0.0001$ & $2491(100.00)$ & 0 & - & $2475(99.36)$ & $16(0.64)$ & $<0.0001$ \\
\hline $\begin{array}{l}\text { Pregnant, } \\
\text { severe heart } \\
\text { disease }\end{array}$ & $<5$ & 0 & - & $<5$ & 0 & - & $<5$ & 0 & - & $<5$ & $0(0.00)$ & - \\
\hline $\begin{array}{l}\text { Immuno- } \\
\text { suppressive } \\
\text { therapy }\end{array}$ & $5285(98.80)$ & $64(1.20)$ & $<0.0001$ & $5299(99.07)$ & $50(0.93)$ & $<0.0001$ & $5346(99.94)$ & $<5$ & - & 5324 (99.53) & $25(0.47)$ & $<0.0001$ \\
\hline $\begin{array}{l}\text { Solid organ } \\
\text { transplant }\end{array}$ & $1228(98.63)$ & $17(1.37)$ & $<0.0001$ & $1230(98.80)$ & $15(1.20)$ & $<0.0001$ & $1244(99.92)$ & $<5$ & - & $1238(99.44)$ & $7(0.56)$ & $<0.0001$ \\
\hline
\end{tabular}




\begin{tabular}{|c|c|c|c|c|c|c|c|c|c|c|c|c|}
\hline & \multicolumn{3}{|c|}{ Confirmed COVID-19 infection } & \multicolumn{3}{|c|}{ COVID-19 hospitalisation } & \multicolumn{3}{|c|}{ COVID-19 ICU admission } & \multicolumn{3}{|c|}{ COVID-19 mortality } \\
\hline & \begin{tabular}{|l|} 
Negative test/ \\
Not tested
\end{tabular} & Positive test & \multirow[b]{3}{*}{$p$ value } & Not admitted & Admitted & \multirow[b]{3}{*}{$p$ value } & Not admitted & Admitted & \multirow[b]{3}{*}{$p$ value } & Alive & Dead & \multirow[b]{3}{*}{$p$ value } \\
\hline & $\mathrm{N}=1,311,723$ & $\mathrm{~N}=3,348$ & & $\mathrm{~N}=1,313,410$ & $N=1661$ & & $\mathrm{~N}=1,314,949$ & $\mathrm{~N}=122$ & & $N=1,314,044$ & $\mathrm{~N}=1027$ & \\
\hline & n (\%) & n (\%) & & n (\%) & n (\%) & & n (\%) & n (\%) & & n (\%) & n (\%) & \\
\hline $\begin{array}{l}\text { Rare } \\
\text { diseases and } \\
\text { IEM }\end{array}$ & $1729(97.96)$ & $36(2.04)$ & $<0.0001$ & $1741(98.64)$ & $24(1.36)$ & $<0.0001$ & $1764(99.94)$ & $<5$ & - & $1744(98.81)$ & $21(1.19)$ & $<0.0001$ \\
\hline $\begin{array}{l}\text { Renal } \\
\text { dialysis }\end{array}$ & $445(93.68)$ & $30(6.32)$ & $<0.0001$ & $457(96.21)$ & $18(3.79)$ & $<0.0001$ & $475(100.00)$ & $0(0.00)$ & - & $468(98.53)$ & $7(1.47)$ & $<0.0001$ \\
\hline
\end{tabular}

Table 2. Crude, population-level COVID-19 outcomes by sociodemographic characteristics, risk category and risk criteria. $\mathrm{N}$ number; IEM inborn errors of metabolism.

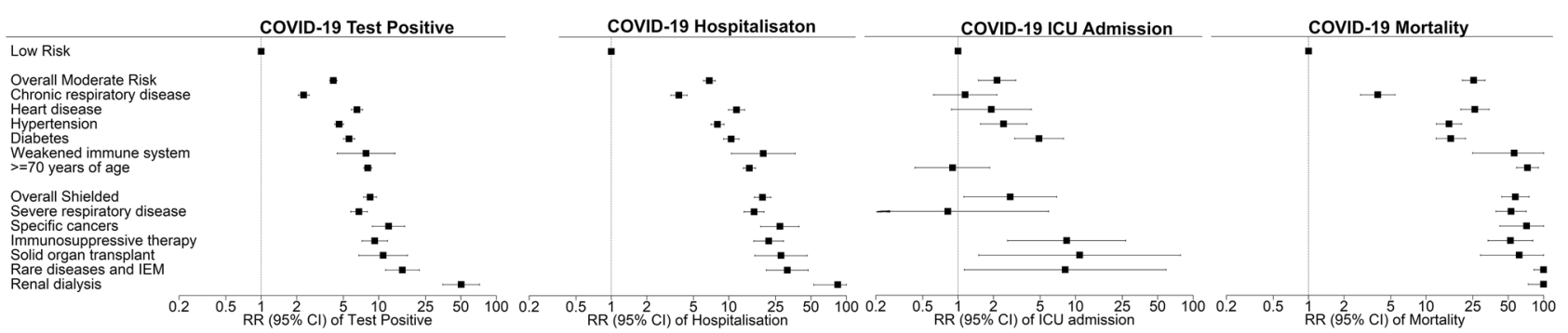

Figure 1. Associations ${ }^{\star}$ between risk categories and risk criteria and population-level COVID-19 outcomes. ${ }^{*}$ Adjusted for sex, deprivation quintile, and other risk categories. RR relative risk; CI confidence interval; IEM inborn errors of metabolism.

One quarter of the population were classified as moderate-risk and not advised to shield. Nonetheless, they were four times more likely to have confirmed infections than the low-risk category, five times more likely to die following confirmed infection and 25 times more likely to die overall, suggesting that, where shielding is employed, the shielding criteria should be expanded. In particular, older age needs to be considered since the elderly are both at high individual risk and contribute significantly to population burden due to their relatively high numbers. A survey of 1695 rheumatology patients reported that the shielded sub-group has slightly lower risk of COVID-19 infection than the non-shielded sub-group (3.0\% vs $4.1 \%$ ) but the difference did not reach statistical significance ${ }^{21}$. In spite of people in the shielded and moderate-risk categories having poorer prognosis, they were less likely to be admitted to ICU following hospitalisation for COVID-19, especially patients $\geq 70$ years. This finding reinforces the importance of protection in those with the worst prognosis.

Our finding that $26.85 \%$ of people satisfied the moderate-risk criteria is consistent with limited existing evidence. A study linking English primary and secondary care records on 3.9 million people reported that $20 \%$ of population satisfied similar criteria ${ }^{22}$. Similarly, analysis of the Global Burden of Diseases Study estimated that $22 \%$ of the global population are at increased risk of severe COVID-19 disease ${ }^{19}$. A USA study using data from the Behavioral Risk Factor Surveillance System reported that $45.4 \%$ of 444,649 adults had one or more of a longer list of morbidities that may be associated with higher risk from COVID- $19^{23}$. Another USA study estimated that $14.2 \%$ of participants in the National Health Interview Survey had more than two-fold risk and $1.6 \%$ had more than tenfold risk ${ }^{24}$.

The evidence on COVID-19 related complications among those classified as high risk, and therefore advised to shield, has mainly come from case series and expert opinion. Case series found higher COVID-19 related complications among organ transplant recipients ${ }^{25,26}$, patients receiving chemotherapy, radiotherapy or immunotherapy for cancer ${ }^{27,28}$, and patients with haematological cancers ${ }^{29}$. Systematic review suggested higher COVID-19 complication risk among COPD patients, but the effect of COPD severity was not investigated ${ }^{30}$. Patients with cystic fibrosis and sickle cell disease were classified as high risk based on expert opinion ${ }^{31,32}$. While pregnant women with COVID-19 were found to have higher risk of poor maternal and perinatal outcomes ${ }^{33,34}$, outcomes were not investigated specifically for pregnant women with heart disease. There was no evidence of worse COVID-19 related complications among patients on immunosuppressants ${ }^{35}$. A large community study in England found strong association between severe asthma (hazard ratio 1.25) and COVID-19 related mortality but did not investigate the risk of COVID-19 infection or hospitalisation ${ }^{36}$.

In common with previous studies ${ }^{37}$, we demonstrated that age was a major individual-level risk factor for death. Additionally, we showed it is important at the population level with $49.55 \%$ of deaths attributable to age $\geq 70$ years. The higher mortality in the elderly was mediated in part by higher case-fatality but they also had a higher incidence of infection, possibly due to transmission within care homes. Lower ICU admissions following hospitalisation for COVID-19 may have contributed to their higher case-fatality. Previous studies have reported that men are at higher risk of COVID-197. Our study demonstrated they are less likely to be tested for COVID-19, have confirmed infection, and be hospitalised. They have comparable overall mortality from COVID-19, due to their lower incidence, but their case-fatality is higher. 


\begin{tabular}{|c|c|c|c|c|c|c|c|c|c|}
\hline & \multicolumn{3}{|c|}{$\begin{array}{l}\text { COVID-19 hospitalisation } \\
\mathrm{N}=3348^{\mathrm{a}}\end{array}$} & \multicolumn{3}{|c|}{$\begin{array}{l}\text { COVID-19 ICU admission } \\
\mathrm{N}=3348^{\mathrm{a}}\end{array}$} & \multicolumn{3}{|c|}{$\begin{array}{l}\text { COVID-19 case-fatality } \\
\mathrm{N}=3846^{\mathrm{b}}\end{array}$} \\
\hline & \multirow{3}{*}{\begin{tabular}{|l|} 
Not admitted \\
$\mathrm{N}=1687$ \\
$\mathrm{n}(\%)$ \\
\end{tabular}} & \multirow{3}{*}{\begin{tabular}{|l|} 
Admitted \\
$\mathrm{N}=1661$ \\
$\mathrm{n}(\%)$ \\
\end{tabular}} & \multirow[b]{3}{*}{$p$ value } & \multirow{3}{*}{\begin{tabular}{|l|} 
Not admitted \\
$\mathrm{N}=3226$ \\
$\mathbf{n}$ (\%) \\
\end{tabular}} & \multirow{3}{*}{\begin{tabular}{|l|} 
Admitted \\
$\mathrm{N}=122$ \\
$\mathrm{n}(\%)$ \\
\end{tabular}} & \multirow[b]{3}{*}{$p$ value } & \multirow{3}{*}{\begin{tabular}{|l|} 
Alive \\
$\mathrm{N}=\mathbf{2 8 1 9}$ \\
$\mathrm{n}(\%)$ \\
\end{tabular}} & \multirow{3}{*}{\begin{tabular}{|l|} 
Dead \\
$\mathrm{N}=1027$ \\
$\mathbf{n}(\%)$ \\
\end{tabular}} & \multirow[b]{3}{*}{$p$ value } \\
\hline & & & & & & & & & \\
\hline & & & & & & & & & \\
\hline $\begin{array}{l}\text { Age group } \\
\text { (years) }\end{array}$ & & & $<0.0001$ & & & $<0.0001$ & & & $<0.0001$ \\
\hline $0-24$ & $97(83.62)$ & $19(16.38)$ & & $116(100.00)$ & 0 & & $116(100.00)$ & $0(0.00)$ & \\
\hline $25-44$ & $410(76.92)$ & $123(23.08)$ & & $520(97.74)$ & $12(2.26)$ & & $526(98.50)$ & $8(1.50)$ & \\
\hline $45-64$ & $553(51.59)$ & $519(48.41)$ & & 990 (92.35) & $82(7.65)$ & & $1003(91.77)$ & $90(8.23)$ & \\
\hline$\geq 65$ & $630(38.65)$ & $1000(61.35)$ & & $1600(98.28)$ & $28(1.72)$ & & $1174(55.83)$ & 929 (44.17) & \\
\hline Sex & & & & & & & & & $<0.0001$ \\
\hline Male & $579(41.12)$ & $829(58.88)$ & $<0.0001$ & $1322(93.96)$ & 85 (6.04) & $<0.0001$ & $1105(68.08)$ & $518(31.92)$ & \\
\hline Female & $1108(57.11)$ & $832(42.89)$ & & $1904(98.09)$ & $37(1.91)$ & & $1717(77.10)$ & $509(22.90)$ & \\
\hline $\begin{array}{l}\text { Deprivation } \\
\text { quintile }\end{array}$ & & & $<0.0001$ & & & 0.29 & & & 0.0004 \\
\hline $\begin{array}{l}1 \text { (most } \\
\text { deprived) }\end{array}$ & $566(43.44)$ & 737 (56.56) & & $1250(96.08)$ & $51(3.92)$ & & $1046(70.34)$ & $441(29.66)$ & \\
\hline 2 & $332(54.07)$ & $282(45.93)$ & & $592(96.57)$ & $21(3.43)$ & & $531(78.90)$ & $142(21.10)$ & \\
\hline 3 & $235(50.98)$ & $226(49.02)$ & & 438 (95.01) & $23(4.99)$ & & $394(72.03)$ & $153(27.97)$ & \\
\hline 4 & $204(51.00)$ & $196(49.00)$ & & 390 (97.50) & $10(2.50)$ & & $341(72.40)$ & $130(27.60)$ & \\
\hline 5 (most affluent) & $353(61.61)$ & $220(38.39)$ & & 556 (97.03) & $17(2.97)$ & & 507 (75.90) & $161(24.10)$ & \\
\hline Risk category & & & $<0.0001$ & & & 0.0001 & & & $<0.0001$ \\
\hline Low & 791 (66.41) & $400(33.59)$ & & $1125(94.54)$ & 65 (5.46) & & $1130(93.08)$ & $84(6.92)$ & \\
\hline Moderate & $828(44.54)$ & $1031(55.46)$ & & $1807(97.20)$ & $52(2.80)$ & & $1485(64.90)$ & $803(35.10)$ & \\
\hline Shielded & $71(23.59)$ & $230(76.41)$ & & 294 (98.33) & $5(1.67)$ & & $204(59.30)$ & $140(40.70)$ & \\
\hline \multicolumn{10}{|c|}{ Moderate risk criteria } \\
\hline $\begin{array}{l}\text { Chronic respira- } \\
\text { tory disease }\end{array}$ & $205(45.35)$ & 247 (54.65) & 0.02 & 439 (97.34) & $12(2.66)$ & 0.29 & $420(88.79)$ & $53(11.21)$ & $<0.0001$ \\
\hline Heart disease & $149(37.72)$ & $246(62.28)$ & $<0.0001$ & $388(98.23)$ & $7(1.77)$ & 0.049 & $330(74.16)$ & $115(25.84)$ & 0.7 \\
\hline Hypertension & $404(42.57)$ & 545 (57.43) & $<0.0001$ & $922(97.26)$ & $26(2.74)$ & 0.10 & $836(78.87)$ & $224(21.13)$ & $<0.0001$ \\
\hline Diabetes & $161(35.31)$ & $295(64.69)$ & $<0.0001$ & $432(94.95)$ & $23(5.05)$ & 0.11 & $398(80.73)$ & $95(19.27)$ & $<0.0001$ \\
\hline $\begin{array}{l}\text { Weakened } \\
\text { immune system }\end{array}$ & $2(16.67)$ & $10(83.33)$ & 0.04 & $12(100.00)$ & 0 & - & $6(50.00)$ & $6(50.00)$ & 0.13 \\
\hline$\geq 70$ years of age & $587(40.37)$ & $867(59.63)$ & $<0.0001$ & $1445(99.45)$ & $8(0.55)$ & $<0.0001$ & $1035(54.10)$ & $878(45.90)$ & $<0.0001$ \\
\hline \multicolumn{10}{|l|}{ Shielding criteria } \\
\hline $\begin{array}{l}\text { Severe respira- } \\
\text { tory disease }\end{array}$ & $33(19.64)$ & $135(80.36)$ & $<0.0001$ & $165(99.40)$ & $<5$ & - & $111(55.78)$ & $88(44.22)$ & $<0.0001$ \\
\hline Specific cancers & $10(25.64)$ & $29(74.36)$ & 0.003 & $39(100.00)$ & 0 & - & $27(62.79)$ & $16(37.21)$ & 0.16 \\
\hline $\begin{array}{l}\text { Pregnant, severe } \\
\text { heart disease }\end{array}$ & - & - & - & - & - & - & - & - & - \\
\hline $\begin{array}{l}\text { Immunosup- } \\
\text { pressive therapy }\end{array}$ & $14(21.88)$ & $50(78.12)$ & $<0.0001$ & $61(95.31)$ & $<5$ & - & $42(62.69)$ & $25(37.31)$ & 0.07 \\
\hline $\begin{array}{l}\text { Solid organ } \\
\text { transplant }\end{array}$ & $2(11.76)$ & $15(88.24)$ & 0.003 & $16(94.12)$ & $<5$ & - & $11(61.11)$ & 7 (38.89) & 0.37 \\
\hline $\begin{array}{l}\text { Rare diseases } \\
\text { and IEM }\end{array}$ & $12(33.33)$ & $24(66.67)$ & 0.06 & $35(97.22)$ & $<5$ & - & $23(52.27)$ & $21(47.73)$ & 0.003 \\
\hline Renal dialysis & $12(40.00)$ & $18(60.00)$ & 0.33 & $30(100.00)$ & 0 & - & $23(76.67)$ & $7(23.33)$ & 0.83 \\
\hline
\end{tabular}

Table 3. Crude COVID-19 outcomes among confirmed cases by sociodemographic characteristics, risk category and risk criteria. N number; IEM inborn errors of metabolism. ${ }^{a}$ Laboratory-confirmed (test-positive) COVID-19 cases. ${ }^{b}$ Clinically-confirmed (test-positive or COVID-19 on death certificate) COVID-19 cases.

This study adds to the existing evidence of the possible effectiveness of a shielding strategy which is largely limited to mathematical modelling of population effects based on assumptions ${ }^{12-20}$. The modelling papers concluded that shielding could potentially reduce deaths and demand for ICU beds, subject to a range of parameters including the transmission rate, proportion shielding, and baseline case-fatality rate, but could not reduce to reproduction rate to below 1 in the absence of other non-pharmaceutical interventions. In Scotland, prior to the introduction of shielding, those individuals subsequently advised to shield accounted for $40 \%$ of COVID19 deaths $^{8}$. However, an unpublished study reported that, following the introduction of shielding, the rate of decline in COVID-19 incidence in the shielding group was comparable to the general population ${ }^{38}$, suggesting 


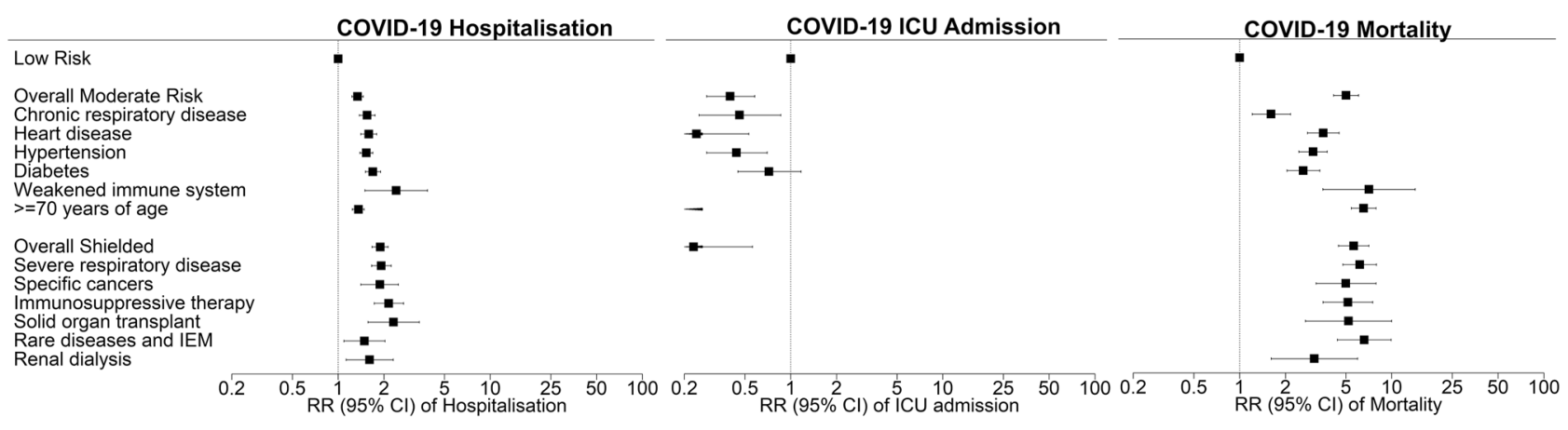

Figure 2. Associations ${ }^{\star}$ between risk categories and risk criteria and COVID-19 outcomes among confirmed cases. ${ }^{\star}$ Adjusted for sex, deprivation quintile, and other risk categories. RR relative risk; CI confidence interval; IEM inborn errors of metabolism.

that the decline was most likely due to the suite of non-pharmaceutical interventions introduced at that time, rather than shielding specifically ${ }^{8,38}$.

Ours was a large-scale, unselected general population study. The data cover a period when shielding was in place. Linkage of family practitioner, laboratory, hospital and death data enabled us to examine a range of COVID-19 outcomes and study a range of exposure variables including the overall risk categories and their individual criteria. The datasets were linked using exact, rather than probabilistic, matching. We were able to adjust for potential sociodemographic confounders. This is important since, in spite of eligibility for Statutory Sick Pay, the financial impact of shielding was greater among people on short, fixed term or zero hours contracts, in self-employment, or unable to work from home, and compliance with shielding was worse in more deprived households ${ }^{39}$. The exposure data were collected prior to the outcomes occurring avoiding potential reverse causation and recall or recording bias. Our analysis of potential risk factors was restricted to those used as criteria for shielding and moderate-risk at the time of the study. We did not have data on ethnicity and rurality and there may be other unmeasured confounders such as exposure to health and social care workers and compliance with restrictions ${ }^{20,40}$. The list of people meeting the shielding and moderate-risk criteria was extracted at the study mid-point; therefore, some people may have been misclassified prior to and after this date.

This is a pragmatic study that evaluates the effectiveness of shielding as delivered and supported in Scotland over the first wave of COVID-19. Shielding was recommended but not monitored or enforced. Forty one percent of people advised to shield in Scotland reported stringently following shielding guidance and $21 \%$ reported they were unable to comply for a variety of reasons such as supporting other household members (e.g. only driver), caring for pets, avoiding domestic abuse, or undertaking essential chores ${ }^{39}$. Many also reported difficulties social distancing within their home due to shared facilities or carer roles. Our findings are representative of Glasgow and Greater Clyde area but may be less so for other areas or countries. In particular, the effectiveness of shielding may be different in countries with different levels of support, monitoring or compliance.

Our findings suggest that attempts to shield those at highest risk have not been as successful as hoped, with those advised to shield experiencing higher rates of infection and death. Since this group was also less likely to be admitted to ICU, protecting them from infection is essential. For shielding to be effective as a population level strategy, the current criteria would need to be expanded, since three-quarters of deaths were associated with moderate-risk criteria for which shielding had not hitherto been recommended. In our study, more than one-quarter of the general population would have needed to be effectively shielded to prevent over $80 \%$ of deaths. Since this is unlikely to be acceptable at a time when governments are under pressure to avoid further lock-downs, shielding is probably best viewed as an intervention to protect individuals, to be used alongside other population-wide interventions such as physical distancing, face coverings and hand hygiene.

\section{Methods}

This was a general population cohort study of all 1.3 million residents of NHS GGC in the West of Scotland between March and May 2020. In Scotland, shielding was recommended from the start of pandemic, throughout the whole study period, until end of July 2020. The Community Health Index (CHI), a unique identifier attached to all Scottish health records, enabled individual-level record linkage of nine databases: Community Health Index (CHI) register, NHS GGC Shielding List, Egton Medical Information Systems (EMIS) and Vision, Electronic Communication of Surveillance in Scotland (ECOSS), Prescribing Information System (PIS), Strathclyde Electronic Renal Patient Record (SERPR), Rapid Preliminary Inpatient Data (RAPID), and death certificates.

The CHI register provided sociodemographic information (age, sex, area socioeconomic deprivation). Deprivation was measured using the Scottish Index of Multiple Deprivation (SIMD), derived from seven domainsincome, education, health, employment, crime, housing, and access to services-and categorised into general population quintiles. ECOSS collects laboratory data on infectious diseases, including test date and result. Albasoft software extract data from the family practitioner electronic health record systems EMIS and Vision, and PIS collects data on medications prescribed by family practitioners. SERPR records data on renal replacement therapy and transplantation. RAPID collects real-time data on hospitalisation, including dates of admission and discharge, and type of ward, and the Scottish Morbidity Record 01 (SMR01) subsequently records the relevant disease codes. Death certificates provide the date and cause of all deaths, whether in-hospital or in the 
community. Follow-up data were available until the end of May 2020, before the shielding recommendation was lifted.

Supplementary Table S1 lists the criteria for the shielded and medium risk categories applied at the time of data extraction. All remaining patients were categorised as low-risk. The Scottish list of high-risk individuals is compiled centrally, and regularly updated, using family practitioner, hospital admission, disease registry and medication data. Family practitioners check the completeness and accuracy of the list before letters, recommending shielding, are sent to patients. The NHS GGC Shielding List we used contains the validated data including the criterion satisfied. We ascertained moderate risk individuals using Albasoft extraction of EMIS and Vision data, and PIS data.

Separate models were conducted by overall risk category (low-risk, moderate-risk or shielded) and by the individual criteria for the moderate-risk and shielded categories. The four general population outcomes investigated were: confirmed COVID-19 infection; COVID-19 related hospitalisation; COVID-19 related ICU admission; COVID-19 related mortality. The three outcomes investigated among those with confirmed infection were: COVID-19 related hospitalisation; COVID-19 related ICU admission; and COVID-19 related case fatality.

Laboratory-confirmed cases were defined as positive PCR test. Clinically-confirmed cases were defined as either positive PCR test or death from COVID-19 without testing. COVID-19 related deaths were defined as International Classification of Diseases 10th revision (ICD-10) code U07.1 or U07.2 recorded on the death certificate. COVID-related hospitalisation was defined as an SMR01 hospitalisation record with an ICD code U07.1 or U07.2 or, for more recent admissions, a RAPID hospitalisation record plus positive PCR test taken between two weeks before and two days after hospitalisation. ICU admission during such hospitalisations was assumed to be COVID-related.

Sociodemographic characteristics were compared by risk category using chi-square tests. Poisson regression models with robust standard errors were used to compare risk ratios (RR) for the shielded and moderate-risk categories referent to the low-risk category. Applying Poisson models to binary outcomes can be subject to an under-dispersion problem. This was overcome by deriving robust 'sandwich' standard errors ${ }^{41}$. The models were run univariately; then adjusted for sex, SIMD quintile, and other risk categories as potential confounders. Age was not included as a covariate because it was a moderate-risk criterion. The models were re-run using the individual criteria for the shielded and moderate-risk categories as the exposure variables, referent to the low-risk category.

Population attributable fractions (PAFs) ${ }^{42}$ were calculated, from prevalence and adjusted RR, to determine the proportion of each outcome that could be attributed to being shielded and moderate-risk, as well as the proportion due to each individual criterion. The PAFs of individual criteria were proportionally calibrated so that their sum equated to the overall PAF of the relevant risk category. PAF confidence intervals were estimated using bootstrapping $(\times 1000)$ taking account of the variance for prevalence and RRs.

Ethical approvals. The study was approved by the NHS GGC Primary Care Information Sharing Group and the NHS GGC Local Privacy Advisory Committee (Reference GSH/20RM005) and was covered by the generic Safe Haven Research Ethics Committee approval (GSH20RM005_COVID_Community). Explicit consent was not feasible to obtain as the data custodians provided us with an anonymised extract of secondary data. Requirement for consent was waived by the Safe Haven Research Ethics Committee, as part of the study approval, on the basis of adequate data protection safeguards including: anonymisation, analysis with a safe haven environment, information governance training, disclosure control and signed agreements. The study has been performed in accordance with the Declaration of Helsinki.

Received: 30 October 2020; Accepted: 9 July 2021

Published online: 27 July 2021

\section{References}

1. Willan, J., King, A. J., Jeffery, K. \& Bienz, N. Challenges for NHS hospitals during covid-19 epidemic. BMJ 368, (2020).

2. Tulloch, O. Shielding People at High Risk of Severe Outcomes from COVID-19. https://opendocs.ids.ac.uk/opendocs/bitstream/ handle/20.500.12413/15286/SSHAPCOVID-19 Brief Shielding.pdf (2020).

3. ACAPS. COVID19 a Global Joint Response. https://www.acaps.org/projects/covid-19 (2020).

4. Swedish Civil Contingencies Agency (MSB). National ban on visiting elderly homes. (2020).

5. Public Health England. Guidance on shielding and protecting people who are clinically extremely vulnerable from COVID-19. (2020).

6. Public Health Scotland. Covid-19 Shielding Programme (Scotland) Rapid Evaluation Full Report. https://www.publichealthscotland. scot/media/2949/covid-19-shielding-programme-scotland-rapid-evaluation-full-report.pdf (2021).

7. Lipsitch, M., Swerdlow, D. L. \& Finelli, L. Defining the epidemiology of Covid-19-Studies needed. N. Engl. J. Med. 382, 1194-1196 (2020).

8. Williams, A. N. Respectful disagreement: The covid-19 shielding list. BMJ 372, (2021).

9. Lochlainn, M. N. et al. Key predictors of attending hospital with COVID19: An association study from the COVID Symptom Tracker App in 2,618,948 individuals. medRxiv 2020.04.25.20079251. https://doi.org/10.1101/2020.04.25.20079251 (2020).

10. Williamson, E. J. et al. Factors associated with COVID-19-related death using OpenSAFELY. Nature 584, 430-436 (2020).

11. Wynants, L. et al. Prediction models for diagnosis and prognosis of covid-19 infection: Systematic review and critical appraisal. BMJ 369, m1328 (2020).

12. Vlas, S. J. de \& Coffeng, L. E. A phased lift of control: a practical strategy to achieve herd immunity against Covid-19 at the country level. medRxiv. https://doi.org/10.1101/2020.03.29.20046011 (2020).

13. Keeling, M. J. et al. Predictions of COVID-19 dynamics in the UK: Short-term forecasting and analysis of potential exit strategies. PLoS Comput. Biol. 17, e1008619 (2021). 
14. McKeigue, P. M. \& Colhoun, H. M. Evaluation of 'stratify and shield' as a policy option for ending the COVID-19 lockdown in the UK. medRxiv. https://doi.org/10.1101/2020.04.25.20079913 (2020).

15. Bunnik, B. Van, Woolhouse, M. \& van Bunnik, B. A. D. Segmentation and shielding of the most vulnerable members of the population as elements of an exit strategy from COVID-19 lockdown. medrxiv. https://doi.org/10.1101/2020.05.04.20090597 (2020).

16. Weitz, J. S. et al. Modeling shield immunity to reduce COVID-19 epidemic spread. Nat. Med. 26, 849-854 (2020).

17. Neufeld, Z., Khataee, H. \& Czirok, A. Targeted adaptive isolation strategy for COVID-19 pandemic. Infect. Dis. Modell. 357-61. https://doi.org/10.1101/2020.03.23.20041897 (2020).

18. Acemoglu, D., Chernozhukov, V., Werning, I. \& Whinston, M. Optimal Targeted Lockdowns in a Multi-Group SIR Model. https:// doi.org/10.3386/w27102 (2020).

19. Clark, A. et al. Global, regional, and national estimates of the population at increased risk of severe COVID-19 due to underlying health conditions in 2020: A modelling study. Lancet Glob. Heal. https://doi.org/10.1016/S2214-109X(20)30264-3 (2020).

20. Davies, N. G. et al. Effects of non-pharmaceutical interventions on COVID-19 cases, deaths, and demand for hospital services in the UK: A modelling study. Lancet Public Heal. https://doi.org/10.1016/S2468-2667(20)30133-X (2020).

21. Cleaton, N. et al. The impact of COVID-19 on rheumatology patients in a large UK centre using an innovative data collection technique: Prevalence and effect of social shielding. Rheumatol. Int. 1-8 (2021).

22. Banerjee, A. et al. Estimating excess 1-year mortality associated with the COVID-19 pandemic according to underlying conditions and age: A population-based cohort study. Lancet https://doi.org/10.1016/s0140-6736(20)30854-0 (2020).

23. Adams, M. L., Katz, D. L. \& Grandpre, J. Population based estimates of comorbidities affecting risk for complications from COVID19 in the US. medRxiv. https://doi.org/10.1101/2020.03.30.20043919 (2020).

24. Jin, J., Agarwala, N., Kundu, P. \& Chatterjee, N. Estimating the size of high-risk populations for COVID-19 mortality across 442 US cities. https://doi.org/10.1101/2020.05.27.20115170 (2020).

25. Pereira, M. et al. COVID-19 in solid organ transplant recipients: Initial report from the US epicenter. Am. J. Transplant https:// doi.org/10.1111/AJT.15941 (2020).

26. Fernández-Ruiz, M. et al. COVID-19 in solid organ transplant recipients: A single-center case series from Spain. Am. J. Transplant https://doi.org/10.1111/AJT.15929 (2020).

27. Zhang, L. et al. Clinical characteristics of COVID-19-infected cancer patients: A retrospective case study in three hospitals within Wuhan, China. Ann. Oncol. Off. J. Eur. Soc. Med. Oncol. https://doi.org/10.1016/J.ANNONC.2020.03.296 (2020).

28. Dai, M. et al. Patients with cancer appear more vulnerable to SARS-COV-2: A multi-center study during the COVID-19 outbreak. Cancer Discov. https://doi.org/10.1158/2159-8290.CD-20-0422 (2020).

29. He, W. et al. COVID-19 in persons with haematological cancers. Leukemia 1-9. https://doi.org/10.1038/s41375-020-0836-7 (2020).

30. Yang, J. et al. Prevalence of comorbidities and its effects in coronavirus disease 2019 patients: A systematic review and meta-analysis. Int. J. Infect. Dis. 94, (2020).

31. Colombo, C. et al. Impact of COVID-19 on people with cystic fibrosis. Lancet Respir. Med. 8, e35-e36 (2020).

32. Rodriguez-Morales, A. et al. Clinical, laboratory and imaging features of COVID-19: A systematic review and meta-analysis. Travel Med. Infect. Dis. https://doi.org/10.1016/J.TMAID.2020.101623 (2020).

33. Di Mascio, D. et al. Outcome of coronavirus spectrum infections (SARS, MERS, COVID 1-19) during pregnancy: A systematic review and meta-analysis. Am. J. Obstet. Gynecol. MFM https://doi.org/10.1016/J.AJOGMF.2020.100107 (2020).

34. Elshafeey, F. et al. A systematic scoping review of COVID-19 during pregnancy and childbirth. Int. J. Gynaecol. Obstet. https://doi. org/10.1002/IJGO.13182 (2020).

35. Minotti, C., Tirelli, F., Barbieri, E., Giaquinto, C. \& D, D. How is immunosuppressive status affecting children and adults in SARSCoV-2 infection? A systematic review. J. Infect. S0163-4453, (2020).

36. The OpenSAFELY et al. OpenSAFELY: Factors associated with COVID-19-related hospital death in the linked electronic health records of 17 million adult NHS patients. medRxiv 2020.05.06.20092999. https://doi.org/10.1101/2020.05.06.20092999 (2020).

37. Ho, F. K. et al. Is older age associated with COVID-19 mortality in the absence of other risk factors? General population cohort study of 470,034 participants. PLoS ONE 15, e0241824 (2020).

38. McKeigue, P. M. et al. Relation of severe COVID-19 in Scotland to transmission-related factors and risk conditions eligible for shielding support: REACT-SCOT case-control study. medRxiv (2021).

39. Delvaux, J. \& Marosi, D. Covid-19 Shielding Programme (Scotland) Impact and Experience Survey. https://www.publichealthsco tland.scot/downloads/covid-19-shielding-programme-scotland-impact-and-experience-survey/?utm_source=websitenews\&utm_ medium=newsitem\&utm_campaign=shieldingsurvey $(2020)$.

40. Besançon, L., Meyerowitz-Katz, G., Chini, E. Z., Fuchs, H. \& Flahault, A. Challenges in determining causality: An ongoing critique of Bendavid et al's "Assessing mandatory stay-at-home and business closure effects on the spread of COVID-19." Eur. J. Clin. Invest. e13599 (2021).

41. Zou, G. A modified Poisson regression approach to prospective studies with binary data. Am. J. Epidemiol. 159, 702-706 (2004).

42. Vander Hoorn, S., Ezzati, M., Rodgers, A., Lopez, A. D., \& Murray, C. J. Estimating Attributable Burden of Disease from Exposure and Hazard Data. Comparative Quantification of health risks: Global and Regional Burden of Disease Attributable to Selected Major Risk Factors. (World Health Organization, 2004).

\section{Acknowledgements}

We are extremely grateful to the following for their support and hard work in extracting and linking the datasets and providing access to them: Charlie Mayor (Data Safe Haven Manager), Alison Hamilton (Safe Haven Project Manager), Jonathan Todd (Head of Information Management), Imran Sadat (Data Manager) and Neil Hillen (Data Analyst).

\section{Author contributions}

F.H. and B.J. contributed equally and are joint-first authors. B.J. and J.P. conceptualised the study, interpreted the data, and wrote the first draft of the manuscript. F.H. analysed the data and wrote the first draft of the manuscript. All other authors interpreted the data and critically revised the manuscript. All authors approved the final submitted version of the manuscript. B.J., F.H., and J.P. serve as the guarantor of the manuscript and accepts full responsibility for the work and/or the conduct of the study, had access to the data, and controlled the decision to publish. The corresponding author attests that all listed authors meet authorship criteria and that no others meeting the criteria have been omitted.

\section{Funding}

No external funding sources. 


\section{Competing interests}

The authors declare no competing interests.

\section{Additional information}

Supplementary Information The online version contains supplementary material available at https://doi.org/ 10.1038/s41598-021-94630-6.

Correspondence and requests for materials should be addressed to J.P.P.

Reprints and permissions information is available at www.nature.com/reprints.

Publisher's note Springer Nature remains neutral with regard to jurisdictional claims in published maps and institutional affiliations.

(c) (1) Open Access This article is licensed under a Creative Commons Attribution 4.0 International License, which permits use, sharing, adaptation, distribution and reproduction in any medium or format, as long as you give appropriate credit to the original author(s) and the source, provide a link to the Creative Commons licence, and indicate if changes were made. The images or other third party material in this article are included in the article's Creative Commons licence, unless indicated otherwise in a credit line to the material. If material is not included in the article's Creative Commons licence and your intended use is not permitted by statutory regulation or exceeds the permitted use, you will need to obtain permission directly from the copyright holder. To view a copy of this licence, visit http://creativecommons.org/licenses/by/4.0/.

(C) The Author(s) 2021 